\title{
POSITIVE CURVATURE AND EIGENFUNCTIONS OF THE LAPLACIAN
}

\author{
HAROLD DONNELLY
}

(Communicated by Mikhail Shubin)

\begin{abstract}
Examples are given of noncompact Riemannian manifolds having nonnegative Ricci cuvature and infinitely many square integrable eigenfunctions for the Laplace operator.
\end{abstract}

\section{INTRODUCTION}

Let $M$ be a noncompact manifold endowed with a complete Riemannian metric. The Laplacian $\Delta$ is a second order elliptic operator depending upon the Riemannian metric. Solutions of $\Delta \phi=-\lambda \phi$ are smooth by elliptic regularity. If $\phi$ is square integrable, then so is its gradient $\nabla \phi$ and moreover $\lambda \int \phi^{2}=-\int \phi \Delta \phi=\int|\nabla \phi|^{2} \geq$ 0 . So $\lambda \geq 0$ and $\lambda=0$ is possible only if $\phi$ is constant and $M$ has finite volume. The question of existence of eigenfunctions with positive eigenvalue depends more delicately upon the geometry of the Riemannian manifold.

We now assume that $M$ has nonnegative Ricci curvature. If the metric of $M$ is rotationally symmetric, then Escobar [5] proved that $\Delta$ has no square integrable eigenfunctions. Rellich identities were employed by Escobar and Freire [6] and the author and Garofalo [4] to show absence of point spectrum. Rotational symmetry need not be assumed. The underlying manifolds include certain perturbations of generalized paraboloids. These methods were subsequently extended by the author [3] to show that there are no square integrable eigenfunctions for Riemannian manifolds with nonnegative Ricci curvature, maximal volume growth, and quadratic curvature decay.

The purpose of the present note is to provide examples where $M$ has nonnegative Ricci curvature and infinitely many square integrable linearly independent eigenfunctions. These examples are doubly warped products which contain embedded submanifolds with cylindrical ends. We begin by presenting some relevant background concerning manifolds with cylindrical ends. Much deeper results concerning the point spectrum for cylindrical manifolds were proved by Christiansen and Zworski [2]. Our examples are given explicitly in the final section of this paper.

Received by the editors November 24, 2006.

2000 Mathematics Subject Classification. Primary 58J50, 58J53.

This author was partially supported by NSF grant DMS-0504729. 


\section{CylindricAl MANifoldS}

Suppose that $X$ is a compact Riemannian manifold. The Laplacian of $X$ has pure point spectrum consisting of eigenvalues $\lambda_{i}$ repeated according to multiplicity. One may form the generalized cylinder $M=R \times X$ with the product metric denoted by $(d r)^{2}+g_{X}$. By employing separation of variables, we decompose the Laplacian of $M$ into a direct sum of ordinary differential operators $\frac{-d^{2}}{d r^{2}}+\lambda_{i}$, acting on $L^{2}(R, d r)$. In particular, the spectrum of the Laplacian is absolutely continuous of finite but nonconstant multiplicity.

We now consider the more general situation where $M=R \times X$ is endowed with a warped product metric $(d r)^{2}+f^{2}(r) g_{X}$ and $f(r)=1$ for $|r|>r_{0}$. Separation of variables now yields the ordinary differential operators $\mathcal{D}_{i}=\frac{-d^{2}}{d r^{2}}+h(r)+\lambda_{i} f^{-2}(r)$. The term $h(r)$ arises from renormalizing the measure on $R$ to Lebesgue measure. In particular, $h(r)=0$ for $|r|>r_{0}$. The essential spectrum of $\mathcal{D}_{i}$ is $\left[\lambda_{i}, \infty\right)$, as in the case where $f$ is identically one for all $r$. However, if $f(r)>1$ for some $r$, then $\mathcal{D}_{i}$ will have point spectrum for all sufficiently large $i$. These eigenvalues of $\mathcal{D}_{i}$ are less than $\lambda_{i}$ and must exist by application of the minimax principle. The crucial point for our purposes is that arbitrarily small perturbations of the product metric yield point spectrum on the warped product. This point spectrum will consist of infinitely many eigenvalues.

Similar considerations apply to manifolds $M$ with a pole and one cylindrical end. In geodesic polar coordinates on $R^{+} \times S^{n-1}$, the metric is of the form $(d r)^{2}+$ $f^{2}(r) g_{X}$. Here $X=S^{n-1}$ and $g_{X}$ is the standard round metric. For cylindrical ends, $f(r)$ is constant for $r>r_{0}$. Separation of variables now yields an ordinary differential operator with a regular singular point at $r=0$, but this poses no serious difficulty. If $f(r)>f\left(r_{0}+1\right)$ for some $r$, the previous argument applies. One deduces that the Laplacian of $M$ has infinitely many eigenvalues.

It is easily checked that the above constructions do not produce manifolds of positive Ricci curvature. In fact, Escobar [5] proved the absence of eigenvalues for rotationally symmetric metrics of positive Ricci curvature.

\section{Doubly WARped products}

We now present our examples of complete noncompact Riemannian manifolds of positive Ricci curvature whose Laplacian has point spectrum. In fact, the point spectrum is of infinite multiplicity and embedded in the continuum. These manifolds $M$ are diffeomorphic to $R^{3} \times S^{2}$. If $r$ is the geodesic distance from the origin in $R^{3}$, then the metric is a doubly warped product on $R^{+} \times S^{2} \times S^{2}$. The metric will be written as $d r^{2}+f_{1}^{2}(r) g_{1}+f_{2}^{2}(r) g_{2}$. One chooses the functions $f_{1}$ and $f_{2}$ to be asymptotically constant with positive limiting values $c_{1}$ and $c_{2}$ as $r \rightarrow \infty$. Moreover, $f_{2}(r)=c_{2}$, for $r>r_{0}$. It is necessary to require a finite value of $c_{2}$, but $c_{1}$ is allowed to be infinite. The limiting values of $f_{1}^{\prime}$ and $f_{1}^{\prime \prime}$ should be zero as $r \rightarrow \infty$.

Separation of variables is applicable as in the case of generalized cylinders. If $\lambda_{i}$ denote the eigenvalues for $S^{2}$ with its standard metric, then the Laplacians of our examples decompose into a direct sum of ordinary differential operators $\mathcal{D}_{i j}=\frac{-d^{2}}{d r^{2}}+h(r)+\lambda_{i} f_{1}^{-2}(r)+\lambda_{j} f_{2}^{-2}(r)$ on the Hilbert space $L^{2}\left(R^{+}, d r\right)$. Our hypotheses about the warping functions ensure that $h(r) \rightarrow 0$ as $r \rightarrow \infty$. The essential spectrum of $\mathcal{D}_{i j}$ is thus $\left[c_{1}^{-2} \lambda_{i}+c_{2}^{-2} \lambda_{j}, \infty\right)$. Suppose that $f_{2}(r)>c_{2}$, for 
some value of $r$. If $\lambda_{j}$ is sufficiently large, then the minimax principle ensures that $\mathcal{D}_{i j}$ has eigenvalues less than $c_{1}^{-2} \lambda_{i}+c_{2}^{-2} \lambda_{j}$. In short, the spectral theory of these doubly warped products is quite similar to the analysis of cylindrical manifolds.

The advantage of the double warping is that positive Ricci curvature may be achieved. Cheeger and Colding [1, Section 8] derived formulas for the Ricci curvature of multiply warped products. Using these formulas, we see that our examples have positive Ricci curvature provided that the following three inequalities hold:

$$
\begin{aligned}
& \text { (i) } \frac{-f_{1}^{\prime \prime}}{f_{1}}-\frac{f_{2}^{\prime \prime}}{f_{2}}>0, \\
& \text { (ii) } f_{1}^{-2}-\frac{f_{1}^{\prime \prime}}{f_{1}}-\left(\frac{f_{1}^{\prime}}{f_{1}}\right)^{2}-2\left(\frac{f_{1}^{\prime}}{f_{1}}\right)\left(\frac{f_{2}^{\prime}}{f_{2}}\right)>0, \\
& \text { (iii) } f_{2}^{-2}-\frac{f_{2}^{\prime \prime}}{f_{2}}-\left(\frac{f_{2}^{\prime}}{f_{2}}\right)^{2}-2\left(\frac{f_{1}^{\prime}}{f_{1}}\right)\left(\frac{f_{2}^{\prime}}{f_{2}}\right)>0 .
\end{aligned}
$$

If $f_{2}$ is constant, these inequalities reduce to $\frac{-f_{1}^{\prime \prime}}{f_{1}}>0$ and $f_{1}^{-2}\left(1-\left(f_{1}^{\prime}\right)^{2}\right)-\frac{f_{1}^{\prime \prime}}{f_{1}}>0$. So it suffices to have $-f_{1}^{\prime \prime}>0$ and $0 \leq f_{1}^{\prime} \leq 1$. Such functions $f_{1}$ exist with $c_{1}$ any positive value or infinity. If $f_{2}$ is perturbed slightly so that $f_{2}(r)>c_{2}$, for some $r$, the required inequalities continue to hold by continuity. Only a small compactly supported perturbation is required to achieve infinite point spectrum.

The inequality (i) represents the radial Ricci curvature. Although our perturbation of $f_{2}$ will introduce some negative sectional curvature, the average radial sectional curvature remains positive because of the warping function $f_{1}$.

A similar doubly warped product exists in four dimensions based upon $R^{+} \times$ $S^{1} \times S^{2}$. It is a perturbation of the product $P \times S^{2}$, where $P$ is a paraboloid. By taking products of these four and five dimensional examples with copies of $S^{2}$, one obtains manifolds $M$ with any dimension greater than or equal to four. These manifolds are noncompact with positive Ricci curvature and their Laplacians have point spectrum of infinite multiplicity.

We close with a remark concerning eigenvalue asymptotics. Let $N(\lambda)$ denote the number of eigenvalues less than $\lambda$. Suppose that $M^{n}=R^{+} \times S^{j} \times S^{2} \times \cdots \times S^{2}$, $j=1,2$, is one of the given examples. Here $n=\operatorname{dim} M \geq 4$. In these examples the warping function for the factor $S^{j}$ may be unbounded while the warping function for all subsequent factors $S^{2}$ is always assumed to be bounded. If the warping function on the first factor $S^{j}$ is unbounded, then $N(\lambda)$ is infinite for sufficiently large finite $\lambda$. If the warping function on the factor $S^{j}$ is bounded and its derivatives decay sufficiently rapidly, then $N(\lambda) \sim a \lambda^{n / 2}$, as $\lambda \rightarrow \infty$. This last estimate is obtained by summing the standard one dimensional formulas [7, p. 272] for Schrödinger operators. Exponential decay for the derivatives of the warping function is more than sufficient.

\section{REFERENCES}

1. Cheeger, J. and Colding, T., On the structure of spaces with Ricci curvature bounded below I, Journal of Differential Geometry 46 (1997), 406-480. MR1484888 (98k:53044)

2. Christiansen, T. and Zworski, M., Spectral asymptotics for manifolds with cylindrical ends, Ann. Inst. Fourier Grenoble 45 (1995), 251-263. MR1324132 (96d:35100)

3. Donnelly, H., Spectrum of the Laplacian on asymptotically Euclidean spaces, Michigan Math. Journal 46 (1999), 101-111. MR1682891 (2000d:58045) 
4. Donnelly, H. and Garofalo, N., Riemannian manifolds whose Laplacians have purely continuous spectrum, Math. Annalen 293 (1992), 143-161. MR1162679 (93c:58219)

5. Escobar, J., On the spectrum of the Laplacian on complete Riemannian manifolds, Comm. Partial Differential Equations 11 (1985), 63-85. MR814547 (87a:58155)

6. Escobar, J. and Freire, A., The spectrum of the Laplacian on manifolds of positive curvature, Duke Mathematical Journal 65 (1992), 1-21. MR1148983 (93d:58174)

7. Reed, M. and Simon, B., Methods of Modern Mathematical Physics, Vol IV, Analysis of Operators, Academic Press (1978). MR0493421 (58:12429c)

Department of Mathematics, Purdue University, West Lafayette, Indiana 47907 